\title{
Barred Galaxies in the Virgo Cluster
}

\author{
Victor Andersen \\ University of Alabama, Department of Physics and Astronomy, \\ Tuscaloosa, AL, 35487-0324
}

\begin{abstract}
.
Spiral galaxies in the Virgo cluster appear to be barred more frequently than galaxies in less dense environments. Tidal forces due to the cluster mass concentration seem to be the most likely mechanism for inducing these bars.
\end{abstract}

\section{Introduction}

The galaxies in the cores of present day galaxy clusters are preferentially found to be elliptical and lenticular galaxies, rather than spiral galaxies which are predominant in lower density regions of the universe (Gisler 1980; Dressler 1980). Observations of clusters at redshifts of $z \sim 0.3-0.4$ show a much higher percentage of spirals in these clusters than in present day clusters (Couch et al. 1994; Dressler et al. 1994a,b). Apparently some environmental effect alters cluster spirals at high redshift beyond recognition by the current day; possible mechanisms include ram pressure sweeping of the interstellar medium by the intra-cluster medium (Gunn \& Gott 1972), galaxy-galaxy collisions (Richstone 1975), or tidal effects due to the gravitational field of the cluster (Merritt 1983). Unfortunately to date, it has been difficult to distinguish observationally between the various possible mechanisms.

Simulations of galaxy-galaxy interactions (Noguchi 1988; Gerin, Combes \& Athanassoula 1990) and interactions of a disk galaxy with a cluster gravitational field (Byrd \& Valtonen 1990) show that both types of interaction can stimulate the formation of bars in galaxies. Thus, the frequency of barred galaxies in a cluster gives some information about the recent interaction history of those galaxies. The purpose of this study is to examine the frequency of barred galaxies in the Virgo cluster as an aid in discriminating between different environmental effects in cluster galaxies.

\section{Sample and Analysis}

The initial sample consisted of all galaxies from the Virgo cluster catalog of Binggeli, Sandage \& Tammann (1985) with $B_{T} \leq 14.0$. Morphological types were taken from the Third Reference Catalogue of Bright Galaxies (RC3) (de Vaucouleurs et al. 1991), and the sample was limited to Hubble types of Sa and later. Galaxies with $R_{25} \geq 2.5$ were excluded from the sample in order to avoid cases where distinguishing between barred and non-barred morphology 

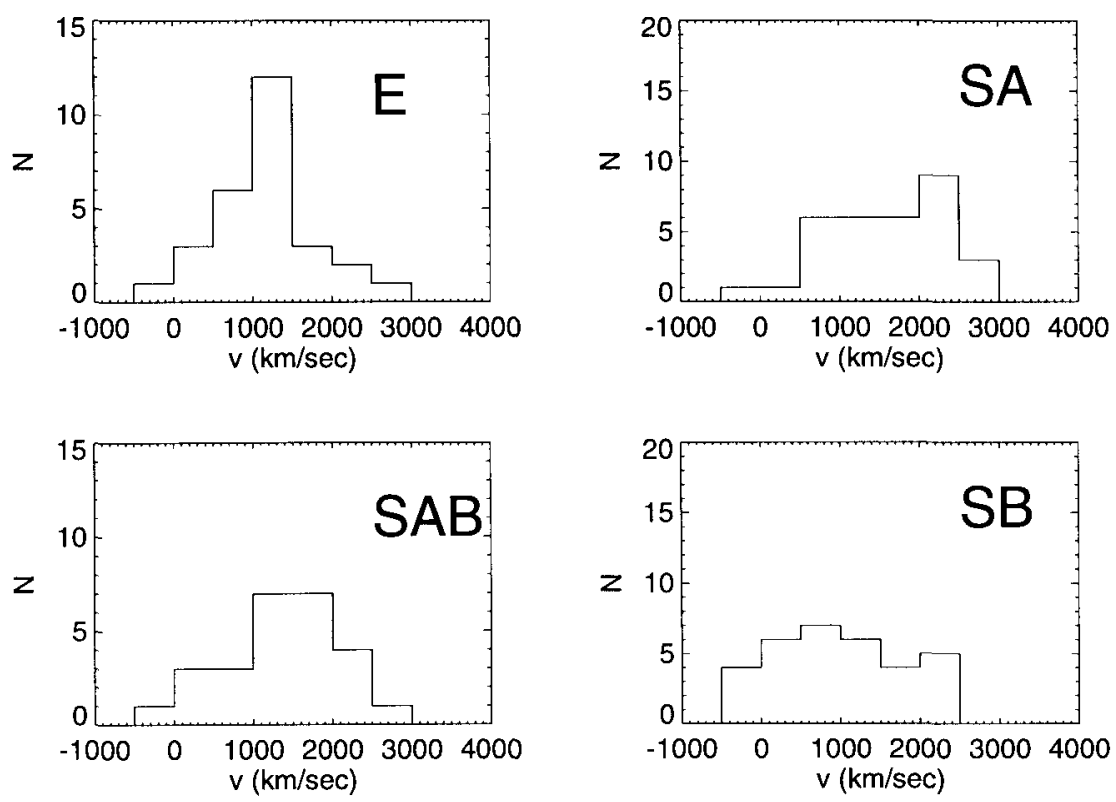

Figure 1. Velocity histograms for the galaxy subsamples.

was difficult. Galaxies which had $v_{\odot} \geq 3000 \mathrm{~km} \mathrm{~s}^{-1}$ were excluded, since it is unlikely that they are actual members of the Virgo cluster. This left a final sample of $32 \mathrm{SA}$ galaxies, $32 \mathrm{SB}$ galaxies, and $26 \mathrm{SAB}$ galaxies.

The primary condensation of the Virgo cluster is centered near the giant elliptical galaxy M87, and contains the bulk of the elliptical and lenticular galaxies in the cluster. The spiral and irregular galaxies are less strongly clumped in the area of M87, although there is a density maximum there. The velocity histogram of the late type galaxies is broader than that of the ellipticals, and shows the presence of multiple velocity peaks. In addition to a peak at the velocity of the early-type component $\left(v_{\odot} \approx 1100 \mathrm{~km} \mathrm{~s}^{-1}\right)$, there are components at $v_{\odot} \approx 2200$ $\mathrm{km} \mathrm{s}^{-1}$ which non-velocity dependent distance indicators place at roughly the distance implied by their recession velocity, and a group with low heliocentric velocities that lie in projection within the core region of the cluster. The TullyFisher relation puts these low velocity galaxies at the distance of Virgo's main condensation, and it has been argued (Tully \& Shaya 1984) that these galaxies are currently falling into the cluster core for the first time.

Given the complicated spatial structure of the Virgo cluster, I chose initially to examine the distribution of barred versus non-barred galaxies in velocity space. An attempt to disentangle the spatial distribution of galaxies belonging only to Virgo's main condensation is described elsewhere (Andersen 1995). Velocity histograms for the E, SA, SAB and SB galaxies are given in Figure 1. To formally test the difference between the distribution of galaxies of different bar strength, the Kolmogorov-Smirnov (KS) test was employed. For the purposes 
of the KS test, the velocities of $\mathrm{SB}$ galaxies were compared to the combined velocities of the SA and SAB galaxies. The KS test finds only a $3 \%$ probability that the velocity distributions of the $\mathrm{SB}$ and $\mathrm{SA}+\mathrm{SAB}$ samples are drawn from the same population. Examination of the velocity histograms show that the difference between the samples is due to the tendency for Virgo spiral galaxies with low heliocentric velocities to be barred.

\section{Summary}

An enhancement is detected in the fraction of barred galaxies in the Virgo cluster, in the sense that the low velocity galaxies thought to be falling into the cluster core for the first time are preferentially barred. Either interactions between galaxies (enhanced because of the increased galaxy density at the cluster center) or a tidal interaction of the galaxy with the cluster gravitational field may explain this result. Galaxy-cluster interactions may be preferred, since all galaxies falling into the cluster should experience the cluster tidal field.

Acknowledgments. Support for this paper is provided by the Alabama Space Grant Consortium, and by NSF EPSCoR grant EHR-9108761. This research has made use of the NASA/IPAC Extragalactic Database (NED) which is operated by the Jet Propulsion Laboratory, California Institute of Technology, under contract with the National Aeronautics and Space Administration.

\section{References}

Andersen, V. 1995, in preparation

Binggeli, B., Sandage, A., \& Tammann, G. A. 1985, AJ, 90, 1681

Byrd, G. \& Valtonen, M. 1990, ApJ, 350, 89

Couch, W. J., Ellis, R. S., Sharples, R. M. \& Smail, I. 1994, Ap.J, 430, 121

de Vaucouleurs, G., de Vaucouleurs, A., Corwin, H. G., Buta, R. J., Paturel, G.

\& Fouqué, P. 1991, Third Reference Catalogue of Bright Galaxies, New York: Springer (RC3)

Dressler, A. 1980, ApJ, 236, 351

Dressler, A., Oemler, A., Butcher, H. R., \& Gunn, J. E. 1994a, ApJ, 430, 107

Dressler, A., Oemler, A., Sparks, W. B. \& Lucas, R. A. 1994b, ApJ, 435, 23L

Gerin, M., Combes, F., \& Athanassoula, E. 1990, A\&A, 230, 37

Gisler, G. R. 1980, AJ, 85, 623

Gunn, J. E. \& Gott, J. R. 1972, ApJ, 176, 1

Merritt, D. 1983, ApJ, 264, 24

Noguchi, M. 1988, A\&A, 203, 259

Richstone, D. O. 1975, ApJ, 200, 535

Tully, R. B. \& Shaya, E. J. 1984, ApJ, 281, 31 